\title{
Perception Enhanced Virtual Environment for Maritime Applications
}

\author{
Damitha Sandaruwan, Nihal Kodikara, Chamath Keppitiyagama and Rexy Rosa
}

\begin{abstract}
This paper presents the development of a realtime perception enhanced virtual environment for maritime applications which simulates real-time six degrees of freedom ship motions (pitch, heave, roll, surge, sway, and yaw) under user interactions, environmental conditions and various threat scenarios. This simulation system consists of reliable ship motion prediction system and perception enhanced immersive virtual environment with greater ecological validity. This virtual environment supports multiple-display viewing that can greatly enhance user perception and we developed the ecological environment for strong sensation of immersion. In this virtual environment it is possible to incorporate real world ships, geographical sceneries, several environmental conditions and wide range of visibility and illumination effects. This system can be used for both entertainment and educational applications such as consol level computer games, teaching \& learning applications and various virtual reality applications. Especially this frame work can be used to create immersive multi user environments.
\end{abstract}

\section{INTRODUCTION}

Perception enhanced virtual environments have been used for naval training, simulating military scenarios and entertainment activities such as computer games. In general all these applications are real-time applications.

There are commercial ship simulation systems with great ecological validity [1] such as Transas [2] and Oceaniccorp [3]. These are extremely expensive, proprietary and closed systems. Users can have versatile and realistic functionalities and users can customize these functionalities up to some extent [4]. However if users try to simulate existing real world then they have enormous restrictions because their real-time ship motion algorithms, integration mechanisms 3D objects such as naval vessels and navigation areas are hidden.

Manuscript received March 22, 2010. This research was funded by the National e-Learning center, Sri Lanka and supported by Department of electrical and electronic Sri Lanka navy.

Damitha Sandaruwan is with the University of Colombo School of Computing, 35, Reid Avenue, Colombo, Sri Lanka 7; Tel: +94 -11- 2581245/ 7( e-mail: dsr@ucsc.cmb.ac.lk).

Nihal Kodikara and Chamath Keppitiyagama are also with the University of Colombo School of Computing, 35, Reid Avenue, Colombo 7, Sri Lanka; Tel: +94 -11-2581245/7(e-mail: ndk@ucsc.cmb.ac.lk, Chamath@ ucsc.cmb.ac.lk)

Rexy Rosa is with the Department of Physics, University of Colombo, Colombo 7, Sri Lanka; Tel:+94 -11- 2584777 (e-mail: rosa@phys.cmb.ac.lk)
The motion of a floating rigid body can be specified by Newton's laws, fluid dynamics and other basic physics, but the motion of a floating rigid body in ocean surface is extremely complicated and difficult to predict [5]. All six possible degrees of freedom (6DOF) in a motion of a ship can be illustrated in Figure 1. Surge, heave, and sway are translational motions. Roll, yaw, and pitch are rotational motions [6]. In a ship simulation system real-time ship motion simulation algorithms perform a major task. CPU efficiency and accuracy of these algorithms are very important because it can affect the performance of the overall system.

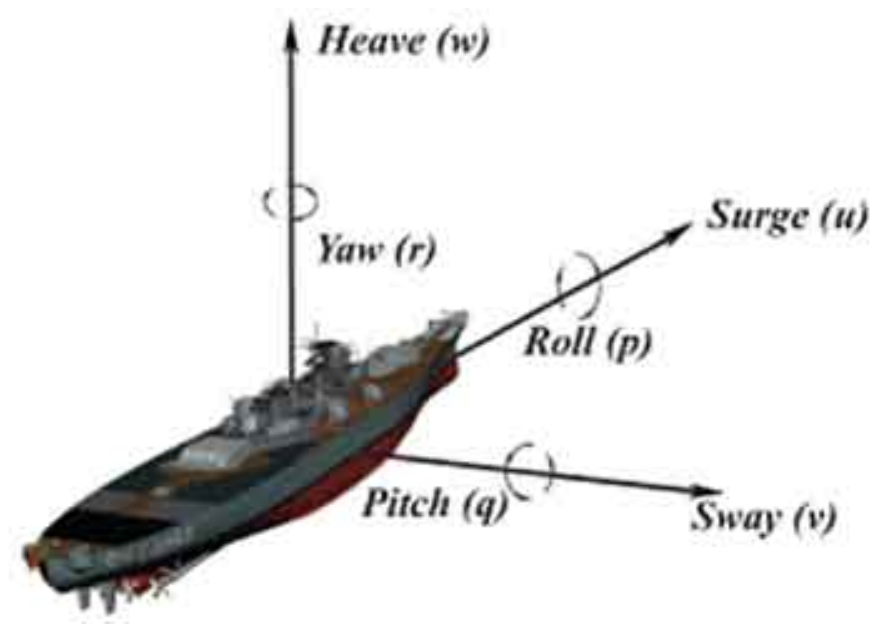

Fig. 1. Six degrees of freedom ship motions

There is no perception enhance virtual environment for marine applications available under an open source license, but there are ship motion prediction systems [7] [8], 3D virtual navigation systems based on deferent rendering engines [9] [10] [11], physics engines [12] [13] and 3D models [14] are available under open source licenses.

We develop our own virtual reality environment for maritime applications with our own algorithms. We used our own six degrees of freedom ship motion prediction system to predict real-time ship motions. We carried out fundamental validations to validate it and obtain satisfactory outcomes. We digitally captured the real world scenarios like naval vessels, moving or fixed targets, and cultural objects and scenes of navigation areas. Subsequently we modeled those sceneries in $3 \mathrm{D}$ space and texturized. We developed a perception enhanced virtual environment with 3000 angle of view based on free and open source rendering engine. We are planning to make this available under an open source license as a total solution for various maritime applications. 


\section{RELATED WORK}

The motion of a floating ship in a sea-way is extremely complicated. However, there are many real-time and none real-time ship motion prediction systems proposed by various researchers and institutes and there are different approaches to predict ship motions [15] [7] [8] [16] [17] [18] [19] [20] [21] [22] [23].

There are a number of physics engines for simulation systems and game developments [12] [13]. Aspects of those physics engines are investigated. The accuracy and computational efficiency of the integrator properties, material properties, stacks, links, and collision detection system are very good but those are not capable of predicting real-time ship motions with respect to the rudder movement, throttle movement and environment conditions [12] .

There are computer games such as ship simulator 2010 [24] and virtual sailor [25]. In these applications users can select various ships, environment conditions and geographical areas associated with their object library and they can adjust principal parameters and properties of those objects. However, users can not incorporate existing real ship or cultural objects to virtual environment and there is no capability to enhance the user perception with multiple display panels.

If we compare the ecological validity of above discussed ship motion simulation methods only a few approaches go towards development of 3D virtual environments [8] [23]. However, those simulation systems do not have multi display screens, physically constructed of Ship Bridge and immersive environment with grater ecological validity. In those applications real-time algorithms were implemented and interact with the virtual environment by using single display panel. However there are other approaches to create immersive environment with grater ecological validity [26] [27]. However they have not discussed the ways of simulating existing geographical areas, naval vessels and their real-time ship motion algorithms.

Alexandre et al. [27] presented a multiple-display Visualization systems that can greatly enhance user perception specially for maritime virtual reality applications. The common approach to provide multiple synchronized views uses a powerful centralized processing unit to support the rendering process on all screens however they used an different approach, the proposed a distributed architecture that supports a flexible and reliable visualization system while giving the users a sensation of immersion with lowend graphics workstations. The proposed system ensured the synchronization of all displayed views. Xiuwen Liu et al. proposed Multi-Projector Tiled Display System for Marine simulator [26]. In their approach is to develop a low-cost multi projector seamless tiled display system commodity hardware and technology for the virtual reality based marine simulators. The main technological problems of the display system are discussed, including geometry distortion adjustment and edge blending. They thought about ecological validity of the virtual environment consequently they constructed real ship bridge and incorporated real ship bridge indicators such as radar and throttle. Experimental results in marine simulator show that this framework is very effective.

There are commercial ship simulation systems [2] [3]. And these simulators provide versatile and realistic ship simulation for maritime teaching, learning, assessment and research. They are capable of predicting real-time ship motions with respect to the rudder movement, throttle movement and environment conditions. However, these systems are extremely expensive and proprietary closed systems. Consequently their ship motion prediction algorithms, constrains and assumptions are unknown. These simulation systems enable users to simulate ship models, cultural objects and illumination effects which are associated with their object library. Users can adjust principal parameters of the objects in the object library [4]. However users can not incorporate existing real ship or cultural objects to the system.

After studying all these approaches we found out that the three degrees of real-time ship motion algorithms proposed by Gatis Barauskis \& Peter Friis-Hansen [7] and the six degrees of real-time ship motion algorithms proposed by Shyh-Kuang Ueng et al. are very effective from different perspectives. However combination of those two approaches can produce more effective and productive real-time six degrees of freedom algorithms. Consequently we combined above mention algorithms [28] and construct immersive virtual environment based on frame works proposed by Alexandre G. Ferreira et al and Xiuwen Liu et al. We tested the new six degrees of freedom real-time ship motion algorithms in immersive environment with greater ethological validity and we discuss the ways of integrating real world geographical areas and real ships.

\section{IMPLEMENTATION OF THE SIMULATION SYSTEM}

\section{A. Overview of the Integrated Simulation System}

We implemented the virtual environment with Matlab [6] and $\mathrm{C}++$. It consists of a ship motion prediction system, Database, rendering engine and a tiled panoramic vision system as illustrated in figure 2. In this virtual environment we use our own computational ship model based on our algorithms so that we can incorporate real world ship data and environment data. The database consists of wind data, ocean wave data, geographical data, naval vessels, moving or fixed targets, and cultural objects. Most of the geographical data and moving or fixed targets are stored in the database in the form of texturized three dimensional mesh models. Selected mesh models are loaded to the rendering engine and virtual camera is placed to view the scenery. Subsequently this virtual camera is driven by the navigational instructions (latitude, longitude, altitude, roll, pitch, and yaw) which are calculated by the computational ship model with respect to vehicle's physical/mechanical properties, user interactions and the environmental conditions. Finally, several virtual cameras are placed in the same virtual environment in separate computers. These virtual cameras maintain deferent directions to create the tiled panoramic vision system. 


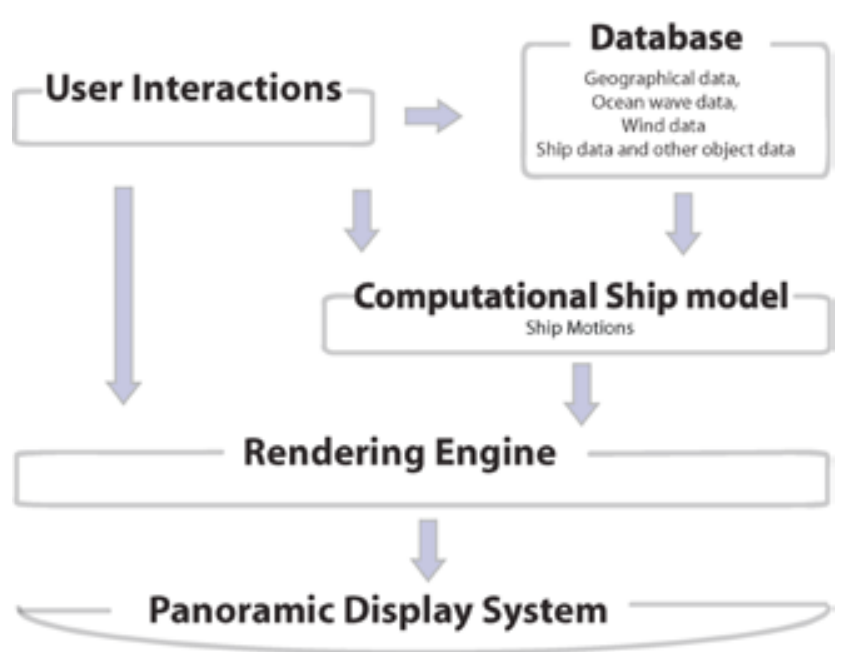

Fig. 2. Structure of the virtual environment

\section{B. Computational Ship Model}

This six degrees of freedom ship motions prediction algorithms respond in real-time to interactions. The computational ship model is derived from the famous linear first order Nomoto ship steering equation [15] [29], linear sway equation, Newton's laws, fluid dynamics and other basic physics [5] [30]. We use multivariable ocean wave model with superposition of sinusoidal functions introduced by Ching-Tang [31]. In this wave model it represents the height of the ocean surface by using the wave amplitude, wave number, wave frequency, direction of the wave and the initial phase. We published detail derivation of these algorithms in the proceedings of CGAT 2009 [28].

This computational ship model consists of two major stages as illustrated in Figure 3. First stage is to compute the ship's position and the orientation in horizontal plane by using ship's physical data, mechanical data and user defined dynamic properties (Rudder, Engine RPM) and the environmental disturbances. The second stage computes the heave, pitch and roll motion by using the outputs of the first stage (ship's position and orientation) and additionally considers the ocean wave model.

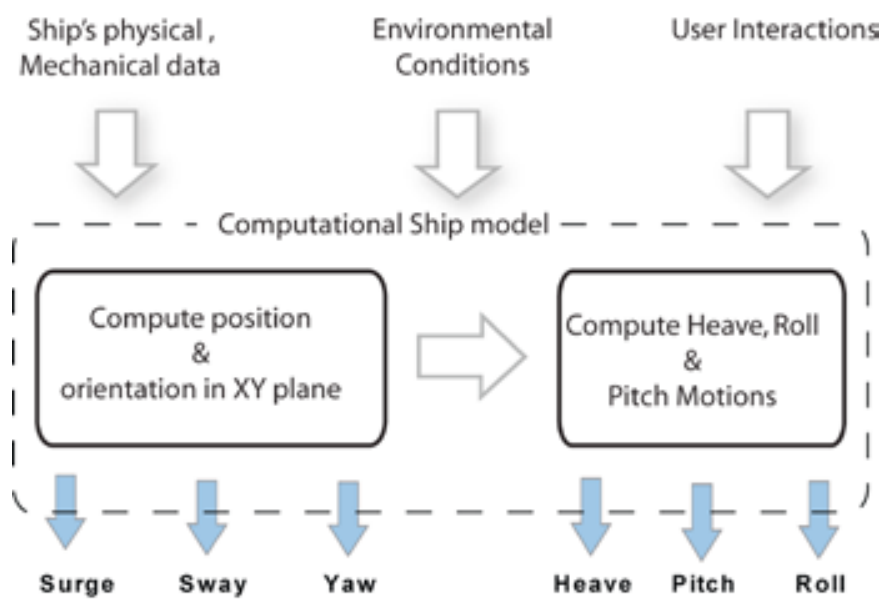

Fig. 3. Structure of the computational ship model.
We use parameters like Nomoto $K$ and Nomoto $\tau$. We can evaluate them with standard maneuvering tests [5] [15] or it is possible to figure out reasonable values from databases such as the Lloyd's register [32].

\section{Validate surge, sway and yaw motions}

Our ship motion prediction algorithms are more suitable for ocean surface vehicles which have approximately box type geometric formations. Our surge, sway and yaw real-time ship motion prediction algorithms were validated with respect to benchmark sea trials of the "Esso Ossaka" [33].

Esso Osaka is a tanker with approximately box type geometric formation as shown in Figure 4.

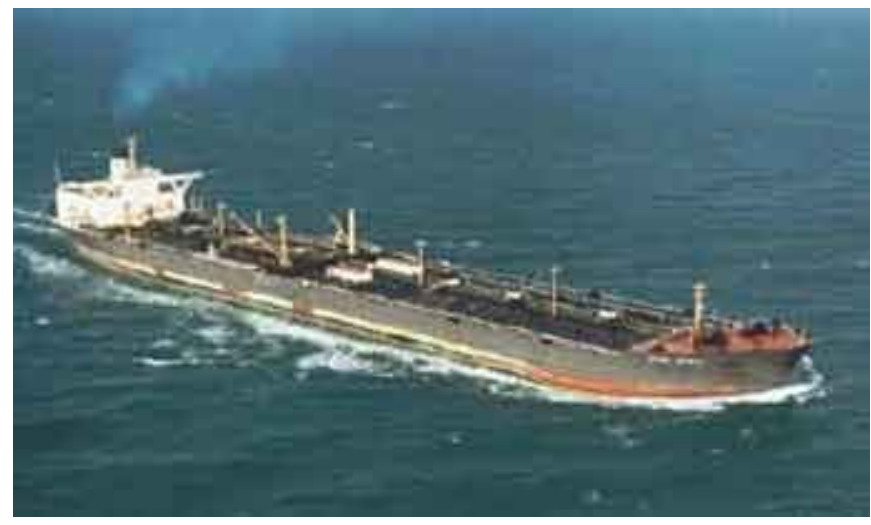

Fig. 4. Esso Ossaka Tanker.

It is an oil tanker that has been used for extensive maneuvering studies in the open literature [34]. Main particulars for the Esso Osaka are Length (between perpendiculars) $-325 \mathrm{~m}$, Beam $-53 \mathrm{~m}$, draft $-21.73 \mathrm{~m}$, Displacement- 4319,400 tonnes. When the propeller rotates at 51 RPM the ship travels at 10 knots in calm water. By using the above mention data and real sea trial data set of Essso Osaka presented by Kevin Mc Taggart [35] [36] we determined the model parameters and simulated the same turning circle under similar conditions in our simulation system. We used MATLAB [37] solvers to solve deferential equations in our real-time algorithms and $2.66 \mathrm{GHz}$ Intel Core 2 Duo iMac to run our computational ship model. Comparison of the real Esso Osaka turning circle and simulated turning circle is shown in the Figure 5.

Comparison of predicted and measured ship motions gives encouraging outcome. We predicted these ship motions under several assumptions as mentioned by Sandariwan et al. [28]. Simulating the real world scenario in a virtual word with similar conditions is not practical there are some problems. We cannot create exactly the similar real world environment conditions such as wave condition in the virtual world. The rate change of the mechanical properties such as the rudder and throttle are extremely difficult to imitate in virtual world. We approximated several 
parameters based on open literature. When we consider all these factors there should be a deviation in simulated results and real world situation.

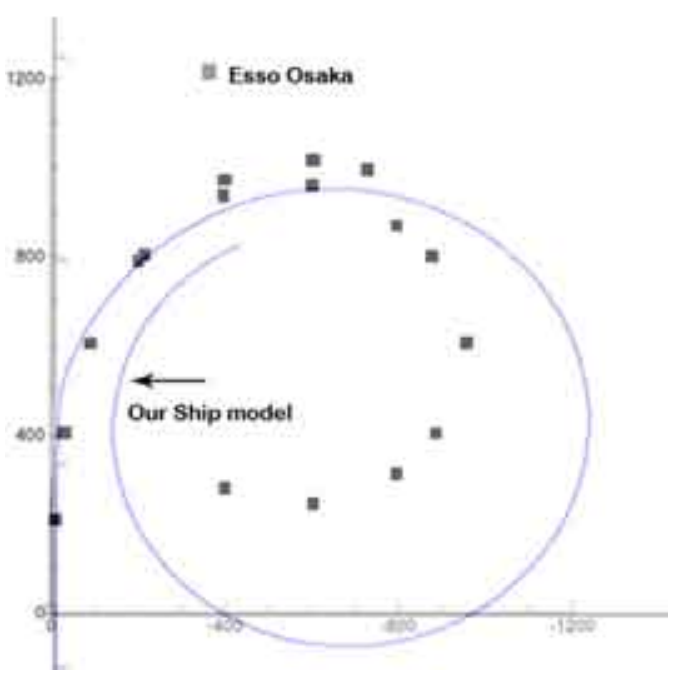

Fig. 5. Validation with respect to benchmark "Esso Ossaka" sea trials

\section{Panoramic Vision System}

Panoramic images and videos are regularly used in various virtual reality applications such as autonomous navigation and virtual walkthroughs [26] [27]. This vision system is based on the clientserver architecture. It supports real-time six degrees of freedom autonomous navigation system with $300^{\circ}$ field of view. The server computer sends the navigational instructions (latitude, longitude, altitude, roll, pitch, and yaw) to the six the client computers as shown in the Figure 6.

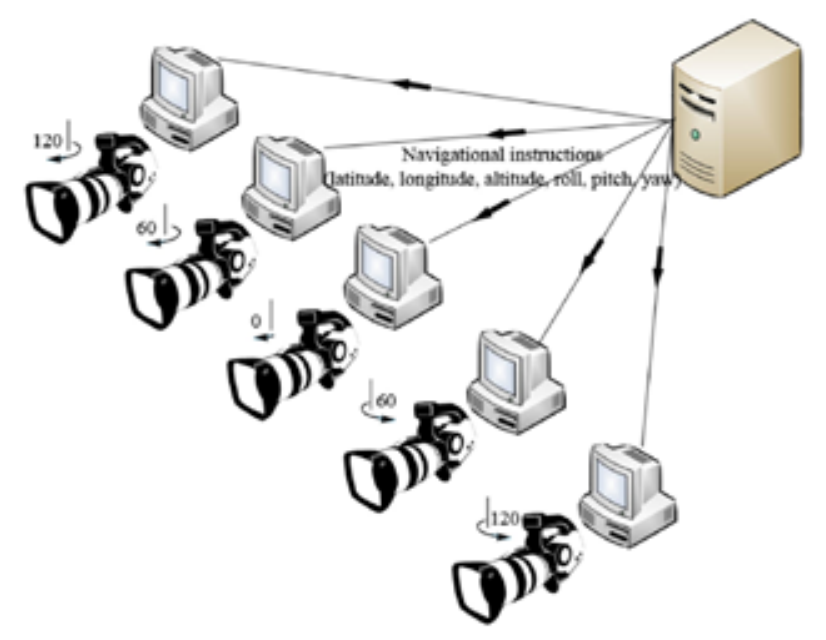

Fig. 6. Structure of the vision system.

In each client computer the same virtual environment is loaded and the position and the orientation values are received from a parental node. Each virtual camera inherits it's position and orientation from the parental node while maintaining 60 degrees with respect to adjacent virtual cameras. To create tile $300^{\circ}$ field of view (FOV) each virtual camera occupies $60^{\circ}$ angle of view [9]. Navigational instructions (latitude, longitude, altitude, roll, pitch, yaw) are send to virtual cameras from the master computer over the network.

We develop a method to synchronize each virtual camera. The sever computer send data set containing latitude, longitude, altitude, roll, pitch, yaw simultaneously to each client computer through net work. Each client PC should place the virtual camera according to the given position and orientation and render the scenery. After completing the rendering process each client machine should send a message to sever computer. Then the sever computer send the next navigational instruction data set after acquiring all six massages from the client computers. This synchronization mechanism reduces the frame rate a little but $2.66 \mathrm{GHz}$ Intel Core 2 Duo iMacs with NVIDIA GeForce $9400 \mathrm{M}$ graphic cards were able to maintain more than 25 Frames per second with a complex scenery.

\section{E. Database}

In this ship simulation system we use our own computational ship models based on our algorithms and constrain so that we can incorporate any computational ship model to our database which satisfy our algorithms. We have developed several six degrees of freedom computational ship models such as benchmark tanker "Esso Ossaka" and offshore patrol vessel quite identical to "Jayasagara" class which has been locally built by the Colombo dockyard [38]. We used Ogre3D rendering engine which has capability of realistic ocean wave rendering [39]. and create real-time interactive environment [40]. We incorporated real world geographical sceneries with cultural objects, moving/fixed targets. Several environmental conditions and wide range of visibility effects such as daytime, dusk and night were incorporated into our database.

We modeled a Sri Lankan harbor and incorporated it into to our database based on the method proposed by Philip Paar et al [41]. We used British admiralty charts "Galle harbour and approaches" as shown in Figure 7 [42] and Google earth [43] images shown in Figure 8 for the 3D modeling.

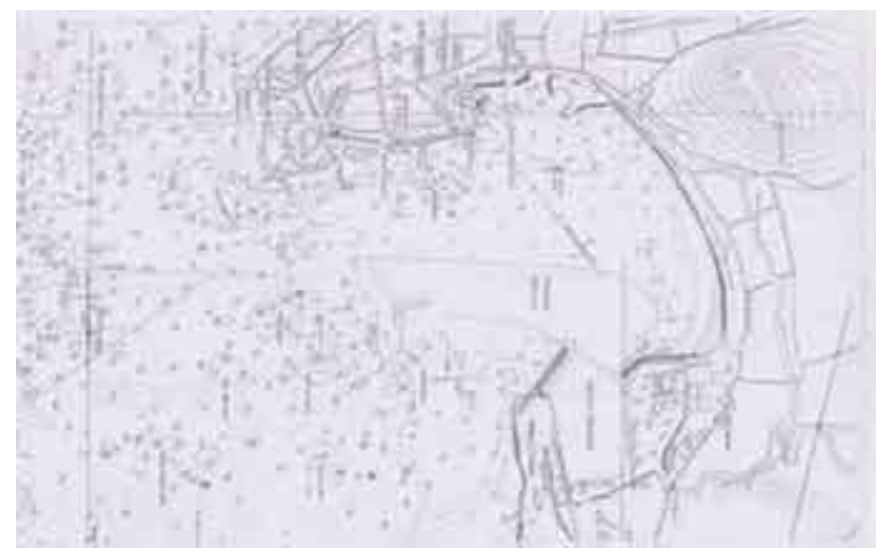

Fig. 7. navigational map of "Galle harbour" 


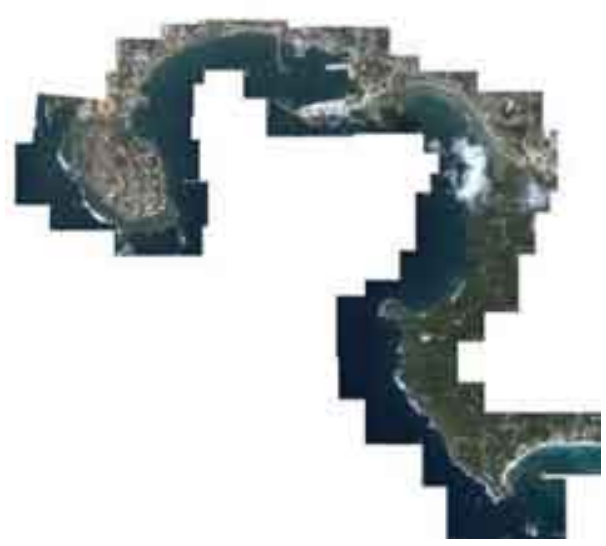

Fig. 8. Top view of the selected harbour.

Subsequently the shore line was finalized and a sequence of digital pictures was taken from the sea while keeping the same distance from the shore line.

The same process was repeated several times to get deferent image sequences with deferent distances from the shore line. Various moving and fixed targets and cultural objects were observed in the sea around the selected harbor. The relative sizes of the observed object were recorded with respect to a selected earth fixed object and digital pictures were taken from different distances.

We used 3D studio max [44] to create mesh models. All naval vessels, moving or fixed targets, and cultural objects, scenes of navigation areas were modeled using 3D studio max. When were modeling the navigation areas and the shore line major objects were modeled with polygonal meshes and the other objects were placed by using the billboards. Throughout this 3D modeling we used appropriate textures to model more realistic scenery. Most of the time we captured the real texture from the digital camera and enhanced by image editing software. Several times we used standard materials from the 3D studio max library. We used OgreMax Scene Exporter [45] to export the modeled scenery from 3D studio max to Ogre 3D. The OgreMax Scene Exporter is a 3DS Max plugin that exports 3DS Max scenes to OgreMax scene files. Finally the virtually created environment is compared with the real environment. The outcome is quite satisfactory level as shown in Figure 9 and Figure 10.

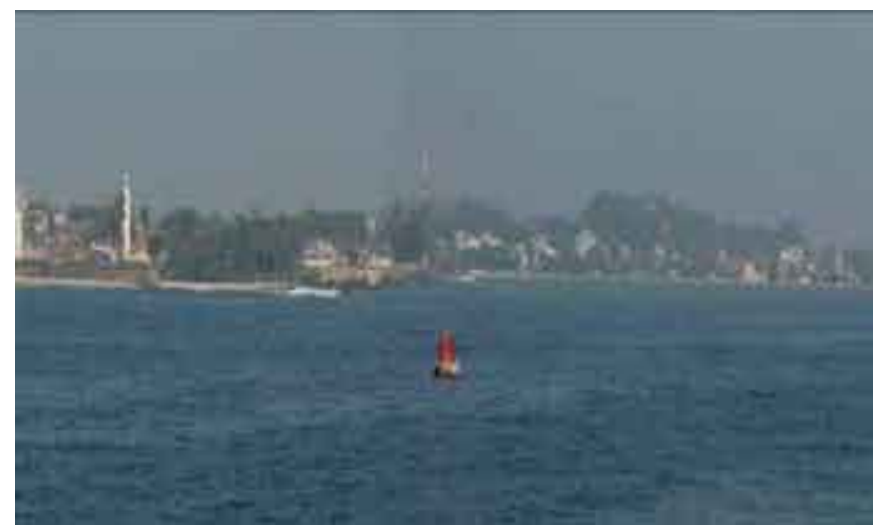

Fig. 9. Real harbor environment

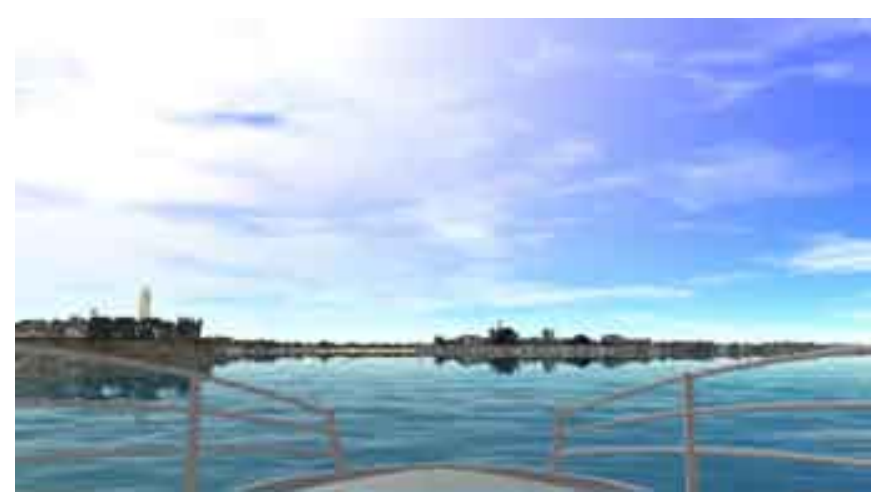

Fig. 10. Virtually created harbor environment

\section{DEVELOPMENT OF IMMERSIVE EN- VIRONMENT}

The immersive environment is composed of 4 standard game PCs - (3 clients and a server) and multi projector seamless tiled display system. It was constructed using three multimedia projectors with 2500 ANSI lumens. This large polygonal screen projected realistic visuals with wide field of view and a real scale bridge was constructed and placed as shown in the figure 11. This brings a sense of seriousness and realism to the user's perception, hence strengthening the ecological validity of the environment.

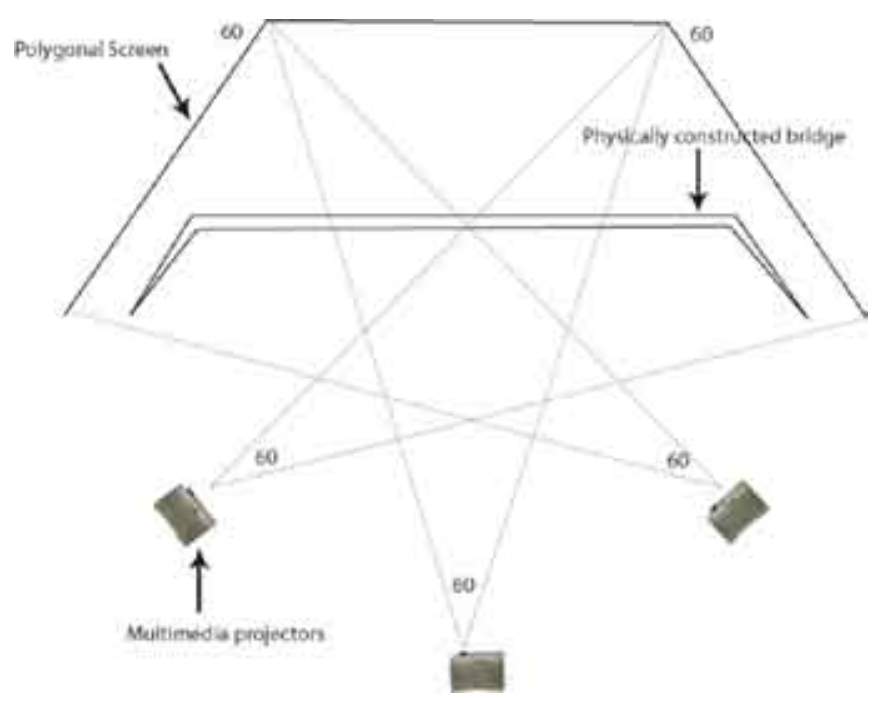

Fig. 11. Bridge and projector arrangement.

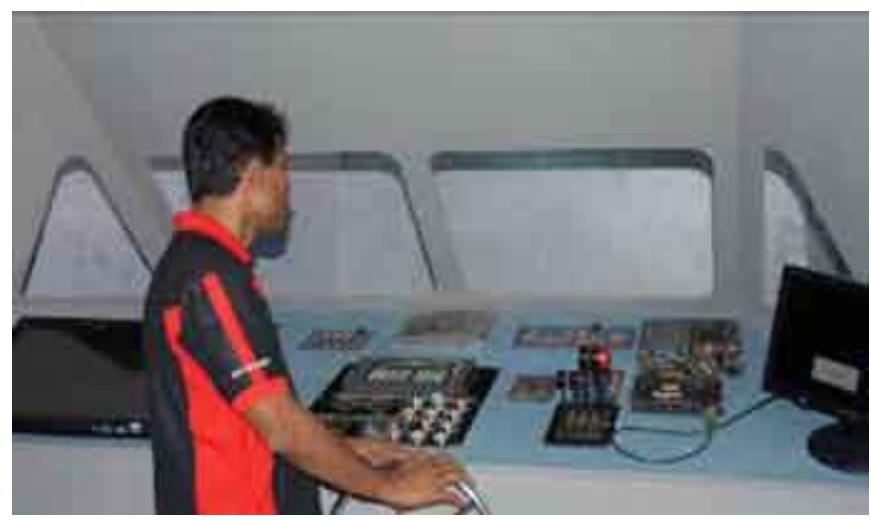

Fig. 12. Physically built ship bridge 
Discarded real equipments were integrated into the bridge. In this case a real throttle and a wheel were interfaced to provide a more realistic virtual environment and a strong sense of immersion as shown in Figure 12. This perception enhanced virtual environment was exhibited at several national and international level exhibitions as shown in figure 13. Large number of people including naval experts, sailors, Navy officers, national and international researchers, game lovers and general public used this immersive environment.

According to the feedback from the naval experts who has more than 15 years of maritime experience with the Sri Lankan navy ships there is a good agreement for surge, sway and yaw motion simulations with respect to the real scenario. There are some divergences in heave, pitch and roll motion simulations with respect to the rendered ocean wave pattern. However we were able to match the desired perception of naval experts by changing several model parameters which are related to heave, pitch and roll motion simulation.

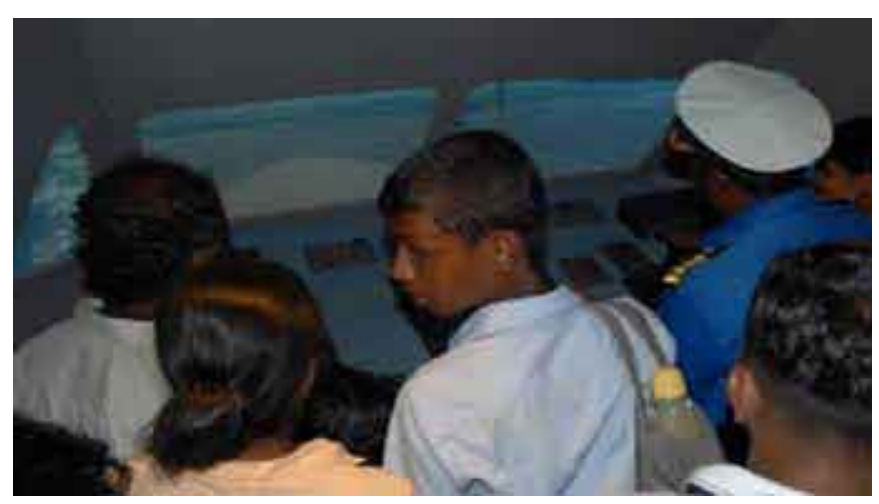

Fig. 13. Virtual environment at an exhibition

\section{SAMPLE MISSION}

A simple mission is developed with the help of the navel experts from the Sri Lanka Navy to match their fundamental navigation lessons with a real ship.

\section{- Mission: Avoid Danger area}

Simulated Ship: offshore patrol vessel ( Ship's length, beam, draft, mass and maximum effective thrust are respectively $48 \mathrm{~m}, 8.6 \mathrm{~m}, 2.2 \mathrm{~m}, 35.6 \mathrm{x}$ $10^{4} \mathrm{Kg}$ and $15.0 \times 10^{4} \mathrm{~N}$ ), Sea State: Beaufort Sea state 3 [5], Present geographical location is given, Mission: Cast off the ship from the present location and proceed to given location and anchorage within given time frame.

Users can use the simulation system and they have to reach the given location within the given time frame. They should find shortest path and avoid the restricted danger area. Several missions were carried out with navel experts to experiment our simulation system and further identify requirements for various difficulty levels.

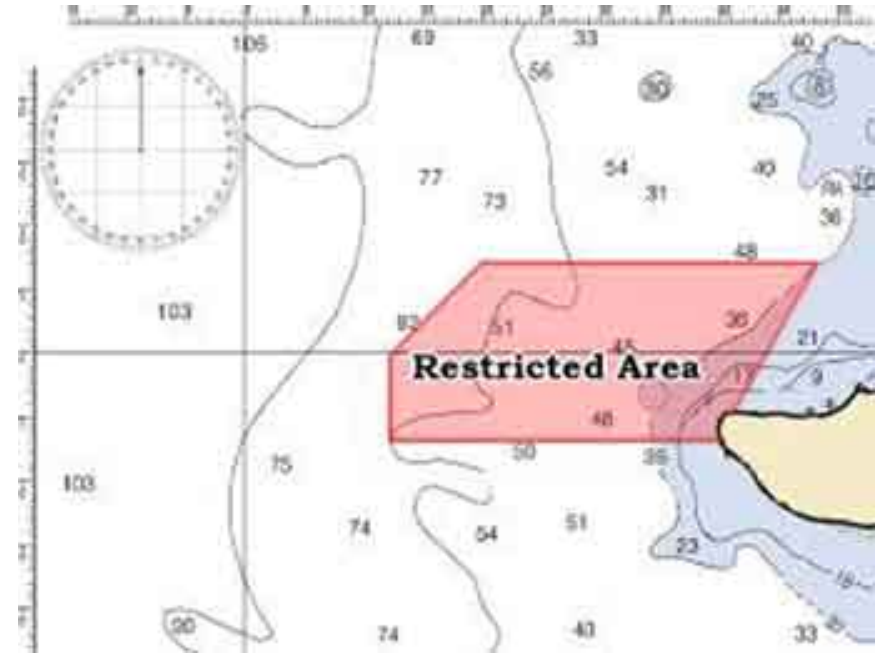

Fig. 20. Geographical map of the navigation area

\section{FUTURE WORK}

This is six degrees of freedom ship simulator but we validated only surge, sway and yaw motions. We have to validate the roll, pitch and heave motions. This will lead to a perfect six degrees of freedom validation of our ship motion simulation. We have to further develop the computational ship model for simulate various kind of modern ships with multiple propellers. Subsequently we have to conduct research to further integrate physics to our simulation system.

Seamless cylindrical tiled display system should be developed to further enhance the trainee's perception. Image stitching and blending algorithms [46] have to be used to get the desired output which is going to be projected on a semi cylindrical. Then the user can get a more immersive maritime experience. Development of a real-time acoustic model for the current simulation system will enhance the ecological validity of the system. In order to use this simulation system in a wide range of missions we have to incorporate more naval vessels, moving or fixed targets, cultural objects, scenes of navigation areas, various environmental effects and conditions to the existing database. We have to conduct research to navigate high polygonal meshes or reduce the polygon count while keeping the visual quality.

\section{CONCLUSION}

Experimental results show that the proposed framework is effective for learning, training and entertainment activities. Open literature show that edge blending and geometry distortion adjustment reduce the frame rate by about $4-10$ frames per second [26]. So this frame work can easily be used for a cylindrical projection system and extendable to a spherical projection system. This display system can be used for both entertainment and educational applications such virtual walkthroughs, ship simulators, flight simulators, military simulations, console level computer games and various virtual reality applications. Specially we can use this frame work for create immersive multi user marine environments. 
In this approach our ship motion prediction algorithms use standard parameters. We can evaluate them with standard maneuvering test or it is possible to figure out reasonable values from the databases such as Lloyd's register. Hence we can simulate existing real ships in our virtual environment. The validation of surge, sway and yaw motion predictions show satisfactory results because there is good agreement with the real world scenarios and the simulated scenarios. This framework can form real-time six degrees of freedom ship motions under various conditions so that it is scalable and configurable. We used our own algorithms in order to customize each and every building block to meet our requirements. We plan to make this ship simulation system available under an open source license so that anyone can customize or further develop it according to their requirements

\section{ACKNOWLEDGMENT}

Our thanks to Department of Electrical and Electronic, Sri Lanka navy and the National e-learning center, Sri Lanka for their collaborative support.

\section{REFERENCE}

[1] Albert A. Rizzo, Todd Bowerly, J. Galen Buckwalter, Dean Klimchuk, Roman Mitura,Thomas D. Parsons., "A Virtual Reality Scenario for All Seasons The Virtual Classroom.” CME 3. January, 2006.

[2] Transas Marine Limited., Transas Simulation Products. [Online] Transas Marine International. [Cited: 06 06, 2010.] http://www. transas.com/products/simulators/.

[3] Oceanic Consulting Corporation., Centre for Marine Simulation. [Online] http://www.oceaniccorp.com/FacilityDetails.asp?id=7.

[4] Transas Marine International., Transas Database editor Model Wizard. Transas. [Online] [Cited: May 06, 2010.] http://www.transas.com/ products/simulators/sim products/navigational/components/tools/

[5] Pinkster, J.M.J. Journée and Jakob., Introduction in Ship Hydromechanics. s.1. : Delft University of Technology, 2002.

[6] Society of Naval Architects and Marine Engineers., Nomenclature for treating the motion of a submerged body through a fluid. New York : The Society of Naval Architects and Marine Engineers, 1950.

[7] Gatis Barauskis, Peter Friis-Hansen., "FAST-TIME SHIP SIMULATOR.” 2007. Safety at Sea 2007.

[8] Shyh-Kuang Ueng, David Lin, Chieh-Hong Liu., "A ship motion simulation system." s.1. : Springer-Verlang, 2008. Virtual Reality (2008). pp. 65-76.

[9] Junker, Gregory., Pro OGRE 3D Programming. s.1. : Apress, 2006. ISBN-13: 978-1590597101.

[10] , Irrlicht Realtime 3D Engine. [Online] [Cited: May 06, 2010.] http:// irrlicht.sourceforge.net/index.html.

[11] Gaming and Simulation Engine. [Online] [Cited: May 06, 2010.] http://www.delta3d.org/.

[12] Adrian Boeing, Thomas Bräunl., "Evaluation of real-time physics simulation systems." Perth, Western Australia : ACM Press, 2007. GRAPHITE 2007. pp. 281-288. ISBN: 9781595939128.

[13] PhysX., physxinfo.com. Popular Physics Engines Comparison Phys X, Havok and $O D E$. [Online] [Cited: May 06, 2010.] http://physxinfo. com/articles/?page_id=154.

[14] , Free Downloads 3D Models. [Online] [Cited: May 06, 2010.] http:// www.3dmodelfree.com/.

[15] Fossen, Thor I., Guidance and Control of Ocean Vehicles. England : Jhon Wiley, 1996. ISBN: 0471941131.

[16] Nils Salvesen, E. O. Tuck and O. Faltinsen., Ship Motion and Sea Loads. New York : Society of Naval Architects and Marine Engineers, 1970.

[17] Journée, J.M.J., "Quick Strip Theory Calculations in Ship Design." Newcastle upon Tyne : s.n., 1992. Practical Design of Ships and Mobile Structures. Vol. 1.

[18] Michael S Triantafyllou, Marc Bodson, Michael Athans., "Real Time
Estimation of Ship Motions Using Kalman Filtering Techniques." IEEE JOURNAL OF OCEANIC ENGINEERING, January 1983, Vols. OE-8, pp. 9-20.

[19] Zhang Xiufeng, Jin Yicheng, Yin Yong, Li Zhihua., "Ship simulation using virtual reality technique." Singapore : ACM, 2004. 2004 ACM SIGGRAPH international conference on Virtual Reality continuum and its applications in industry. pp. 282 - 285 . ISBN:1-58113-884-9.

[20] J. M. Cieutat, J. C. Gonzato, P. Guitton., "A New Efficient Wave Model for Maritime Training Simulator." Budmerice, Slovakia : IEEE Computer Society Washington, DC, USA, 2001. 17th Spring conference on Computer graphics. p. 202 . ISBN 0-7695-1215-1.

[21] Alain Fournier, William T. Reeves., "A simple model of ocean waves." ACM SIGGRAPH Computer Graphics, s.l. : ACM New York, NY, USA, 1986, Issue 4, Vol. 20, pp. 75 - 84 . ISSN:0097-8930.

[22] X Zhao, R Xu, C Kwan., "Ship-motion prediction: algorithms and simulation results.” 2004. ICASSP “04- IEEE International Conference on Acoustics, Speech, and Signal Processing. ISBN: 0-7803-8484-9.

[23] Ching-Tang Chou, Li-Chen Fu., "VR-based Motion Simulator for Dynamics Ship on Six Degree-of-Freedom Platform.” Roma, Italy : s.n., 2007. ICRA International Conference. SBN: 1-4244-0601-3.

[24] , Ship Simulator Extremes. [Online] [Cited: May 06, 2010.] http:// www.shipsim.com/.

[25] Virtual Sailor. [Online] [Cited: May 06, 2010.] http://www.hangsim. com/vs/.

[26] Xiuwen Liu, Cui Xie, Yicheng Jin, Yong Yin., "Construct LowCost Multi-Projector Tiled Display System for Marine Simulator." Hangzhou, China : s.n., 2006. 16th International Conference on Artificial Reality and Telexistence (ICAT'06). pp. 688-693. ISBN: 07695-2754-X.

[27] Alexandre G. Ferreira, Renato Cerqueira, Waldemar Celes Filho, Marcelo Gattass., "Multiple Display Viewing Architecture for Virtual Environments over Heterogeneous Networks." s.1. : IEEE Computer Society Washington, DC, USA, 1999. XII Brazilian Symposium on Computer Graphics and Image Processing. pp. 83 - 92 . ISBN:0-76950481-7.

[28] Damitha Sandaruwan, Nihal Kodikara, Chamath Keppitiyagama, Rexy Rosa., "Realtime Ship Motion Prediction System." s.l. : Research Publising. Computer Games, Multimedia and Allied TechnologyCGAT 09. ISBN 978-981-08-3165-3.

[29] Chen, Ching-Yaw Tzeng and Ju-Fen., "Fundamental Properties of Linear Ship Steering Dynamic Models.” Journal of Marine Science and Technology, 1997, Vol. 7.

[30] Tristan Perez, Mogens Blanke., Mathematical Ship Modeling for Control Applications. 2002.

[31] Fu, Ching-Tang Chou and Li-Chen., "Ships on Real-time Rendering Dynamic Ocean Applied in 6-DOF Platform Motion Simulator." Taichun, Taiwan : s.n., 2007. CACS International Conference.

[32] Lloyd's Register., Lloyd's Register. [Online] [Cited: May 06, 2010.] http://www.lr.org.

[33] Auke Visser's International Esso Tankers site., Esso Osaka - (19731985). [Online] [Cited: May 06, 2010.] http://www.aukevisser.nl/inter/ id427.htm.

[34] The Specialist Committee on Esso Osaka., "Final Report and Recommendations to the 23rd ITTC." 2002. 23rd International Towing Tank Conference.

[35] Mc Taggart, Kevin., Improved Maneuvering Forces and Autopilot Modelling for the ShipMo3D Ship Motion Library. s.1. : Defence R\&D Canada - Atlantic, 2008. DRDC Atlantic TM 2008-162.

[36] McTaggart, Kevin., Simulation of Hydrodynamic Forces and Motions for a Freely Maneuvering Ship in a Seaway. s.l. : Defence R\&D Canada - Atlantic, 2005. DRDC Atlantic TM 2005-071.

[37] The MathWorks, Inc., The MathWorks. [Online] [Cited: May 06, 2010.] http://www.mathworks.com/.

[38] , Jayasagara class patrol craft. [Online] [Cited: May 06, 2010.] http:// en.wikipedia.org/wiki/Jayasagara_class_patrol_craft.

[39] Object-Oriented Graphics Rendering Engine. [Online] [Cited: May 06, 2010.] http://www.ogre3d.org/.

[40] Philip Greenwood, Jesse Sago, Sam Richmond, Vivi Chau., "Using game engine technology to create real-time interactive environments to assist in planning and visual assessment for infrastructure." Cairns, Australia : s.n., 2009. 18th World IMACS / MODSIM Congress.

[41] by Philip Paar, Katy Appleton, Malte Clasen , Maria Gensel , Simon Jude , Andrew Lovett., "Interactive Visual Simulation of Coastal Landscape Change." 2008. Digital Earth Summit on Geoinformatics. 
[42] , British admiralty charts. [Online] [Cited: May 06, 2010.] http://www. mdnautical.com/britishadmiraltycharts.htm.

[43] Satelight Image Source. [Online] [Cited: May 06, 2010.] http://earth. google.com/.

[44] Autodesk., The Autodesk 3D Studio Max Documentations. [Online] [Cited: May 06, 2010.] http://usa.autodesk.com/.

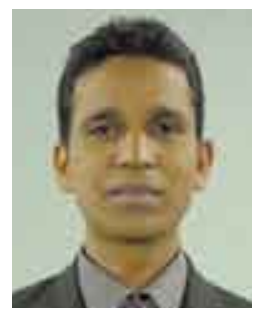

Damitha Sandaruwan received his B.Sc from the University Of Colombo (Faculty of Science). He is currently doing his M.Phil research and working as a media officer at the University of Colombo School Of Computing (UCSC). His research interests include real-time simulations, Mathematical modeling and 3D graphics.

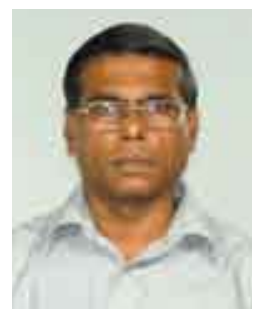

Nihal Kodikara is a Senior Lecturer attached to the University of Colombo School Of Computing. He obtained his B.Sc. from the University of Colombo and M.Sc and Ph.D. in Computer Science from the University of Manchester, U.K. He is presently the Head of the Department of Information Systems Engineering and the Head of Post Graduate Studies at the UCSC. His research interests include Computer Graphics, Computer Vision and e-Governance.
[45] , The Ogremax Documentations. [Online] [Cited: May 06, 2010.] http://www.ogremax.com/.

[46] V Rankov, R J Locke, R J Edens, B Vojnovic., "An algorithm for image stitching and blending.” Proc SPIE (2005). 2005, Vol. 5701, pp. 190-199.

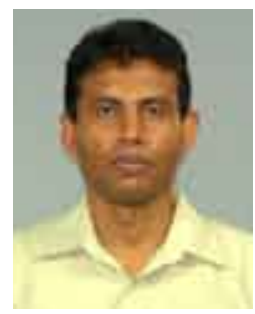

Chamath Keppitiyagama obtained his B.Sc from the University of Colombo and M.Sc and Ph.D in Computer Science from the University of British Columbia (UBC) in Vancouver. He is currently working as a Senior Lecturer at the University of Colombo School Of Computing (UCSC). He is one of the coordinators of the Master's program at UCSC. He has research interests in Distributed Computing, Computer Networks, Operating Systems and Sensor Networks.

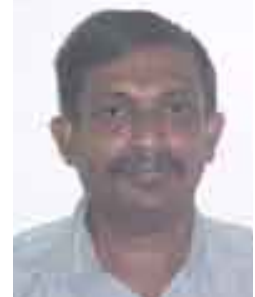

Rexy Rosa is a Senior Lecturer attached to the University Of Colombo Faculty Of Science. He has obtained his B.Sc. from the University of Colombo and M.Sc and Ph.D from the University of Pittsburgh, U.S.A. He is presently the Head of the Department of Physics University of Colombo. His research interests include Gamma Ray Spectroscopy and Physics Education. 